\title{
Estado de nutrición de los refugiados guatemaltecos menores de seis años
}

Liliana Ruiz-Arregui, Lic. en N utr., M. en M.S., (1) Pilar Torre-Medina-Mora, Lic. en N utr., M. en C., (2)

Juan Manuel Castro-Albarrán, M. en C., ${ }^{(3)}$ Herlinda Madrigal-Fritsch, Lic. en N utr., M.S.P.(4)

\section{Ruiz-Arregui L, Torre-Medina-Mora $P$, Castro-Albarrán JM, Madrigal-Fritsch $\mathbf{H}$. Estado de nutrición de los refugiados guatemaltecos menores de seis años. Salud Publica Mex 1998;40:172-180.}

\begin{abstract}
Resumen
Objetivo. Analizar los cambios del estado de nutrición de niños guatemaltecos menores de seis años, refugiados en México entre 1982 y 1996, así como describir los diferentes programas de ayuda alimentaria que se pusieron en marcha. Material y métodos. Se realizó un análisis comparativo de cuatro estudios antropométricos transversales llevados a cabo en Chiapas (1982 y 1989), Q uintana Roo (1989) y Campeche y Q uintana Roo (1996). Se obtuvieron prevalencias de desnutrición según los indicadores peso/edad y talla/edad. Se calculó la razón de momios de la prevalencia y los intervalos de confianza al $95 \%$. Se describen los programas dirigidos a mejorar la nutrición y salud de la población durante el periodo. Resultados. En 1982 privaban altas tasas de desnutrición y de mortalidad entre estos niños refugiados. En 1989, después de su reubicación en Campeche y en Q uintana Roo, había mejorado considerablemente la situación tanto de los reubicados como de los que permanecieron en Chiapas. Esa situación prevaleció hasta 1996. Conclusiones. Los programas emprendidos fueron eficaces para controlar la emergencia y prevenir nuevas situaciones de crisis. Sin embargo, sólo lograron sostener a la población refugiada en condiciones similares a las prevalentes en la población indígena mexicana.
\end{abstract}

Palabras clave: estado nutricional; refugiados/G uatemala; infante; ayuda alimentaria; México

\author{
Ruiz-Arregui L, Torre-Medina-Mora $P$, \\ Castro-A lbarrán JM, Madrigal-Fritsch $\mathbf{H}$. \\ Nutritional status of Guatemalan \\ refugees under six years. \\ Salud Publica Mex 1998;40:172-180.
}

\begin{abstract}
A bstract
Objective. To determine changes in the nutritional status of $G$ uatemalan refugee children under six years of age, who lived in Mexico between 1982 and 1996, under various nutrition-related programs implemented during that period. Material and methods. A comparative analysis of 4 crosssectional anthropometric studies performed in Chiapas (1982, 1989), Q uintana Roo (1989) and Campeche and Q uintana Roo in 1996. Malnutrition prevalence by weight/ age and height/age indicators was calculated. 0 dds ratio and $95 \%$ confidence intervals were estimated. The implemented programs to improve nutritional status and health conditions are described. Results. In 1982, high rates of malnutrition and mortality prevailed among refugee children. In 1989 some had been transferred to C ampeche and Q uintana Roo and others stayed in Chiapas. Their situation had improved and remained so until 1996. Conclusions. The implemented programs were successful to control the emergency and prevent new crises. However they only managed to maintain similar conditions for the refugees as those which prevail among the Mexican indians.
\end{abstract}

Key words: nutritional status; refugees/Guatemala; child; food relief; Mexico

(1) Investigadora el D epartamento de Vigilancia Epidemiológica, Instituto N acional de la N utrición Salvador Zubirán (IN N SZ), México.

(2) Profesora de la Licenciatura en N utrición, Universidad Autónoma Metropolitana-X ochimilco (UAM-X ochimilco), Investigadora del D epartamento de Estudios Experimentales, IN N SZ, México.

(3) Profesor-investigador de la Maestría en Medicina Social, UAM-X ochimilco, México.

(4) Jefa del Departamento de Vigilancia Epidemiológica, IN N SZ, México.

Fecha de recibido: 10 de julio de 1997 • Fecha de aprobado: 23 de enero de 1998

Solicitud de sobretiros: Mtra. Liliana Ruiz Arregui. Cerro del Gallo 17, colonia Pedregal de San Francisco,

Delegación Coyoacán, 04320 México, D.F. Correo electrónico: Iruiz@ quetzal.innsz.mx 
E n diciembre de 1996, con la firma de los acuerdos de paz entre la Unidad Revolucionaria Nacional Guatemalteca (URNG) y el gobierno del presidente Alvaro Arzú, parece haber llegado a su fin un largo proceso de inestabilidad política y deterioro social en Guatemala. Tras 14 años de refugio de miles de guatemaltecos en México, el escenario ofrece lo que se designa como soluciones definitivas al fenómeno de los refugiados: el retorno al país de origen o la asimilación al país receptor.

La presencia de los guatemaltecos en México se explica en el contexto de la crisis económica, política social y militar de Centroamérica en los años ochenta, que generó el desplazamiento de más de un millón de personas, tanto al interior de los países como hacia el exterior. Particularmente en Guatemala, tras el golpe de Estado que colocó en el gobierno al general Efraín Ríos Montt en marzo de 1982, se instauró una doctrina de seguridad nacional que se tipifica como terrorismo de Estado. El resultado fue que más de 100000 personas de origen guatemalteco llegaron a México, acogidas por "la tradicional política mexicana de respeto al derecho de asilo y refugio" ${ }^{*, 1}$

Así, en 1981, en la zona fronteriza del estado de Chiapas, se inició el arribo de guatemaltecos que, en marzo de 1982, adquirió características masivas y se detuvo hasta finales de 1984. Otros guatemaltecos que ingresaron al país llegaron hasta los centros urbanos de varias regiones, adquiriendo la característica de migrantes económicos. ${ }^{2}$

Para marzo de 1984, la Comisión Mexicana de Ayuda a Refugiados (COMAR), organismo del gobierno federal, reconocía la existencia de 92 "campamentos" localizados en los municipios fronterizos del estado de Chiapas, con una población total de 46000 refugiados guatemaltecos. Esos asentamientos iban desde pequeños grupos de cinco familias, hasta el mayor con una población de 4000 habitantes. Más de $90 \%$ de los refugiados eran campesinos de origen indígena, de la etnia maya-quiché. ${ }^{\ddagger}$

Los campamentos en Chiapas fueron objeto de frecuentes hostigamientos por parte del ejército guate-

\footnotetext{
* Alvarez J. Los refugiados y desplazados en Centroamérica. Algunos datos al 30 de septiembre de 1983. Washington, D.C.: Organización Panamericana de la Salud, 1983 (mimeo). United States Senate (Staff Report). Refugee problems in Central America. Washington, D.C.: U.S. Government, 1984. Documento no publicado.

₹ Comisión Mexicana de Ayuda a Refugiados. Informe de actividades. Comisión de Ayuda a Refugiados. Comitán: Secretaría de Gobernación, 1984 (mimeo).
}

malteco por lo que, con los argumentos de la seguridad nacional y la dificultad para una asistencia adecuada en lugares dispersos, en 1984 el gobierno mexicano decidió reubicar a los refugiados en los estados de Campeche y Quintana Roo. Para finales de 1985, cerca de 18500 refugiados habían sido trasladados; 12 500, a dos grandes asentamientos en el estado de Campeche, y 6 000, a otros dos, de Quintana Roo. El resto de los refugiados permaneció en 64 campamentos del estado de Chiapas.

Los nuevos asentamientos se localizaron en terrenos nacionales y fueron planeados para contar con viviendas familiares, terrenos para la producción agropecuaria, infraestructura básica de agua potable, electricidad, caminos, clínicas, escuelas, mercados, iglesias, bodegas y canchas deportivas. Todas las viviendas tuvieron el mismo tamaño y distribución, con dormitorios, cocina y traspatio separados. El financiamiento estuvo a cargo del Alto Comisionado de las Naciones Unidas para Refugiados (ACNUR).*

A lo largo de los 14 años de refugio de los guatemaltecos en México, los autores de este trabajo participaron en el diseño, la ejecución y la evaluación de los programas de ayuda, así como en las evaluaciones antropométricas realizadas a la población, de lo que surge el interés de realizar un análisis global del proceso.

Por lo anterior, los propósitos de este trabajo son los siguientes: a) describir la magnitud de la desnutrición en los menores de seis años y los cambios ocurridos en el periodo estudiado, $\mathrm{y} b$ ) reseñar los programas de ayuda alimentaria que se desarrollaron para esta población.

\section{Material y métodos}

Se trata del anáisis comparativo de cuatro encuestas antropométricas transversales realizadas a la población refugiada guatemalteca menor de seis años durante su estancia en México. Se presentan dos tipos de anáisis: el primero muestra la comparación de las prevalencias de desnutrición encontradas en tres momentos diferentes que abarcan todo el periodo $(1982,1989$, y 1996), con el que se pretende describir el fenómeno de manera general. El segundo, establece la compa-

\footnotetext{
* Comisión Mexicana de Ayuda a Refugiados. Los refugiados guatemaltecos en México, 1982-1988. México, D.F.: Secretaría de Gobernación, 1988. Documento no publicado.

Comisión Mexicana de Ayuda a Refugiados. Informe de actividades en los estados de Chiapas, Campeche y Quintana Roo. México, D.F.: Secretaría de Gobernación, 1985 (mimeo).
} 
ración de la encuesta efectuada en 1989 a la población que permaneció en Chiapas, con la realizada el mismo año entre los reubicados en el estado de Quintana Roo.

\section{Características de cada estudio}

1. Chiapas 1982 (CH82). Es la primera valoración antropométrica que se hizo a esta población, en el momento de su llegada a México. Se evaluó a todos los menores de seis años $(n=712)$ del campamento Puerto Rico, en el municipio de Ocosingo. La evaluación formaba parte de un programa de ayuda alimentaria a grupos vulnerables en el que se incluía el diagnóstico y el tratamiento de los desnutridos. Se seleccionó este campamento por ser el de mayor tamaño y densidad de población (4 000 personas, en ese momento cerca de $20 \%$ del total de los refugiados), y por la magnitud y severidad de los problemas de salud que se presentaban. Asimismo, en su selección influyó el hecho de considerar que sus condiciones eran similares a las de los otros asentamientos de la zona. Las variables observadas fueron el peso y la fecha de nacimiento, con las que se obtuvo el índice peso para la edad. Las fuentes para este análisis fueron informes internos, una tesis y una publicación. ${ }^{3-5}$

2. Quintana Roo 1989 (QR89). Se evaluó al total de la población menor de seis años $(n=1478)$ de los cuatro asentamientos ubicados en el estado: Maya Balam, Kuchumatan, Los Lirios y La Laguna. El objetivo del estudio fue describir la situación general, a fin de establecer modificaciones a los programas de ayuda alimentaria. Las variables que se observaron fueron el peso, la talla y la fecha de nacimiento; con éstas se construyen los índices de peso para la edad y talla para la edad utilizados aquí. Las fuentes son informes internos de Naciones Unidas. ${ }^{*}$

3. Chiapas 1989 (CH89). Se seleccionó una muestra aleatoria por conglomerados de 1182 niños menores de seis años distribuida proporcionalmente entre las zonas geográficas y los estratos (según el tamaño de la población), en 35\% de los 119 asentamientos ubicados en las zonas de Comalapa, Trinitaria y Margaritas. Como en el caso anterior, el objetivo del estudio fue describir la situación

\footnotetext{
* Torre P, Avila A. Reporte sobre la situación nutricional de la población refugiada guatemalteca en el estado de Quintana Roo (informe de trabajo). México, D.F.: INNSZ/ACNUR, 1989. Documento no publicado.
}

general para sustentar la modificación de los programas de ayuda. Las variables y los índices son los mismos que para el CH89. Las fuentes son los informes y la base de datos.*

4. Campeche y Quintana Roo 1996 (CAQR96). Se seleccionó una muestra de 415 niños en edad prescolar, de manera aleatoria, mediante muestreo multietápico, sistemático y con probabilidad proporcional al tamaño de los campamentos, incluyendo los siete asentamientos ubicados en ambos estados: Santo Domingo, Maya Tecum, Quetzal Edzna, Los Laureles, Maya Balam, Kuchumatan y La Laguna. ${ }^{\ddagger}$ El objetivo del estudio fue realizar un informe para la COMAR, bajo la perspectiva de las soluciones definitivas. Las variables estudiadas son: el peso y la edad para obtener el índice peso para la edad. Las fuentes de información son las bases de datos. $\$$

A continuación se presenta la distribución de la población estudiada por edad y sexo de cada uno de los estudios (cuadro I).

Para la primera parte del análisis se seleccionó el índice peso para la edad, mientras que para la segunda, en el caso de QR89 y CH89, se estudiaron el peso y la talla para la edad. El patrón de referencia en todos los estudios fueron las tablas del National Center of Health Statistics. Los criterios de clasificación, de acuerdo con el porcentaje de los índices en relación con el percentil 50, son los siguientes:

1. Peso para la edad, clasificación de Gómez. ${ }^{6}$

Normal $>90 \%$

1er. grado $90-76 \%$

2o. grado $75-61 \%$

3er. grado $\leq 60 \%$

* Comité Técnico Interinstitucional de Nutrición para la Comisión Mexicana de Ayuda a Refugiados. Encuesta nutricional realizada en 36 asentamientos de refugiados guatemaltecos en el estado de Chiapas (informe de trabajo). México, D.F.: Comisión Mexicana de Ayuda a Refugiados, ACNUR, 1989. Documento no publicado.

Madrigal H, Ruiz-Arregui L, Del Maso A et al. Informe de la evaluación del estado de nutrición de los refugiados guatemaltecos asentados en los campamentos de Campeche y Quintana Roo. México, D.F.: INNSZ, 1996. Documento no publicado.

₹ El campamento Los Lirios, que se menciona en Quintana Roo 1989, ya no existía en 1996; su población se distribuyó entre los tres campamentos restantes.

$\S$ Madrigal H. Protocolo para la evaluación del estado de nutrición de los refugiados guatemaltecos, en los campamentos de Campeche y Quintana Roo. México, D.F.: INNSZ, 1996. Documento no publicado. 


\section{Cuadro I \\ Muestras estudiadas POR EDAD}

\begin{tabular}{lrrrr} 
Estudio & \multicolumn{4}{c}{ Edad en meses } \\
\cline { 2 - 5 } & $0-11$ & $12-35$ & $36-71$ & Total \\
CH82 & 144 & 256 & 312 & 712 \\
\hline CH89 & 280 & 537 & 626 & 1443 \\
\hline Q R89 & 332 & 293 & 801 & 1426 \\
\hline CAQ R96 & 23 & 125 & 267 & 415
\end{tabular}

Fuente: elaboración propia con base en datos de las encuestas realizadas a los refugiados guatemaltecos en $\mathrm{CH} 82, \mathrm{CH} 89, \mathrm{Q}$ R89 Y CAQ R96

2. Talla para la edad

Normal $>95 \%$

Pequeños 94-85\%

Achaparrados $\leq 84 \%$

Las mediciones antropométricas se realizaron previa estandarización de los encuestadores. El equipo utilizado fue similar en todos los casos: básculas de reloj con capacidad de $25 \mathrm{~kg}$ y con precisión de $100 \mathrm{~g}$ e infantómetros y estadímetros con capacidad de 130 $\mathrm{cm}$ y $200 \mathrm{~cm}$, respectivamente, y precisión de $1 \mathrm{~mm}$.

Las variables independientes contempladas son el año o lugar del estudio, y la edad y el sexo de los menores, codificados de la siguiente manera:

1. Edad: a) 0 a 11; b) 12 a 35, y c) 36 a 71 meses.

2. Lugar y año del estudio: CH82; QR89; CH89, y CAQR96.

Se compararon las prevalencias de desnutrición mediante el índice peso para la edad, por grupos de edad y totales (estratificadas por edad), de CH82, QR89, y CAQR96, tomando este útimo año como referencia.

Se establecieron las diferencias en la prevalencia de desnutrición según los índices peso para la edad y talla para la edad, por grupos de edad y en población total (estratificada por edad) entre los refugiados asentados en Chiapas y Quintana Roo en el año de 1989.

Tanto para la estimación cruda, como para la estratificada, se calculó la razón de momios de la prevalencia (RMP) y se obtuvieron los intervalos de confianza al 95\% (IC 95\%).

Para el procesamiento de los datos se usaron los paquetes SPSS versión 6.0 para Windows, Anthropo y EPIINFO versión 5.0.

\section{Resultados}

Los cuadros II y III permiten apreciar las diferencias en la prevalencia de desnutrición, por grupos de edad y grados de desnutrición según la clasificación de Gómez, en tres diferentes momentos. El cuadro II muestra de manera general la diferencia entre una situación nutricional de emergencia en CH82 (con características de brote epidémico de desnutrición) y una situación de estabilidad en QR89 y CAQR96 con prevalencias de desnutrición muy similares a las de la población indígena mexicana. ${ }^{78} \mathrm{Al}$ comparar al total de los menores de seis años de CH82 con los de CAQR96 se observa que los primeros tenían aproximadamente 47 y cuatro veces mayor riesgo de padecer desnutrición de III y II grados, respectivamente. En relación con la probabilidad de presentar estado de nutrición normal y desnutrición de I grado se da una relación a la inversa, con cifras significativamente menores en $\mathrm{CH} 82$ (RMP $=0.20$ y 0.44 , respectivamente) comparado con CAQR96. Entre QR89 y CAQR96 no se observan diferencias significativas.

En el mismo cuadro se aprecian las variaciones en el estado nutricional de acuerdo con los grupos de edad. En los tres grupos de menores, se observa en $\mathrm{CH} 82$ un riesgo mayor de presentar desnutrición de II grado que en CAQR96 (RMP $<12$ meses $=7.1,12$ a 35 meses $=3.9$ y 36 a 71 meses $=3.8)$, mientras que en la de III grado sólo se encontraron diferencias significativas en el grupo de 36 a 71 meses con una RMP de 16.3. En CAQR96 no hubo ningún niño menor de 36 meses con desnutrición de III grado. En relación con QR89, el único grupo en el que se observaron diferencias significativas fue en el de 12 a 35 meses, con un riesgo 1.77 veces mayor de padecer desnutrición de II grado respecto a CAQR96.

La situación es inversa en cuanto a desnutrición de I grado y estado de nutrición normal; en la primera se observa un aumento significativo de las prevalencias de CH82 a CAQR96 en los grupos de 12 a 35 y 36 a 71 meses (RMP $=0.35$ y 0.46$)$; en el segundo, el incremento se presenta en los tres grupos de edad (RMP $<12=0.20,12$ a $35=0.12$ y 36 a $71=0.26)$ y, solamente, en el de 12 a 35 meses al comparar QR89 con CAQR96 $(\mathrm{RMP}=0.50)$.

En el cuadro III se compara el riesgo de presentar desnutrición de algún grado para cada uno de los años en las poblaciones totales y por grupos de edad. De manera general se observa que en $\mathrm{CH} 82$ existía un alto riesgo de que la población padeciera desnutrición, destacando la de II grado con una RMP de 11.8, y siguiéndole la de I grado con 7.6 y la de III grado, que en $\mathrm{CH} 82$ es la única que representa un riesgo significativo, con 1.57. En QR89 y CAQR96 solamente la probabilidad de padecer desnutrición de I grado fue significativa, con una RMP de 2.8 en el primero y 4.7 en el segundo. En el anáisis por grupos de edad se observa que el de 


\section{Cuadro II \\ Estado nutricional de la población guatemalteca refugiada menor de Seis años, POR GRUPos de edad, SEgÚN EL INDICAdOR PESO PARA LA EdAd. Comparación POR ESTUdio (CH82, QR89Y CAQR96)}

\begin{tabular}{|c|c|c|c|c|c|c|c|c|c|c|c|c|}
\hline \multirow[t]{2}{*}{ Estudio } & \multicolumn{3}{|c|}{ Normal } & \multicolumn{3}{|c|}{ I grado } & \multicolumn{3}{|c|}{ II grado } & \multicolumn{3}{|c|}{ III grado } \\
\hline & $\%$ & RMP & IC $95 \%$ & $\%$ & RMP & IC $95 \%$ & $\%$ & RMP & IC $95 \%$ & $\%$ & RMP & IC $95 \%$ \\
\hline \multicolumn{13}{|l|}{$<$ de 12 meses } \\
\hline $\mathrm{CH} 82$ & 15.3 & 0.20 & $0.07-0.55$ & 34.0 & 0.67 & ns & 40.3 & 7.08 & $1.52-45.50$ & 10.4 & indef. & \\
\hline Q R89 & 55.4 & 1.36 & ns & 33.1 & 0.64 & ns & 9.0 & 1.04 & $\mathrm{~ns}$ & 2.4 & indef. & ns \\
\hline CAQR96 & 47.8 & 1.00 & - & 43.5 & 1.00 & - & 8.7 & 1.00 & - & 0.0 & 1.00 & - \\
\hline
\end{tabular}

\begin{tabular}{|c|c|c|c|c|c|c|c|c|c|c|c|c|}
\hline $\mathrm{CH} 82$ & 3.5 & 0.12 & $0.05-0.27$ & 30.8 & 0.35 & $0.22-0.56$ & 49.7 & 3.94 & $2.32-6.72$ & 16.0 & indef. & \\
\hline OR89 & 13.7 & 0.50 & $0.29-0.88$ & 51.2 & 0.82 & ns & 30.7 & 1.77 & $1.04-3.03$ & 4.4 & indef & ns \\
\hline CAQR96 & 24.0 & 1.00 & - & 56.0 & 1.00 & - & 20.0 & 1.00 & - & 0.0 & 1.00 & - \\
\hline
\end{tabular}

\begin{tabular}{lrlcccccccccc}
$\begin{array}{l}36 \text { a } 71 \text { meses } \\
\text { CH82 }\end{array}$ & 5.8 & 0.26 & $0.14-0.47$ & 41.3 & 0.46 & $0.33-0.66$ & 47.1 & 3.77 & $2.54-5.61$ & 5.8 & 16.29 & $2.28-329.7$ \\
\hline Q R89 & 22.0 & 1.11 & ns & 60.1 & 0.99 & ns & 16.0 & 0.81 & ns & 1.9 & 5.08 & ns \\
\hline CAQR96 & 20.2 & 1.00 & - & 60.3 & 1.00 & - & 19.1 & 1.00 & - & 0.4 & 1.00 & -
\end{tabular}

\begin{tabular}{lrccccccccccc} 
Total & & & & & & & & & & & & \\
CH82 & 6.9 & 0.20 & $0.12-0.30$ & 36.1 & 0.44 & $0.34-0.57$ & 46.6 & 3.97 & $2.91-5.36$ & 10.4 & 46.85 & indef. \\
\hline Q R89 & 28.1 & 0.93 & ns & 52.0 & 0.91 & ns & 17.4 & 1.07 & ns & 2.5 & 11.06 & indef. \\
\hline CAQR96 & 22.9 & 1.00 & - & 58.1 & 1.00 & - & 18.8 & 1.00 & - & 0.2 & 1.00 & -
\end{tabular}

ns $=$ no significativo

indef. $=$ indefinido

$\mathrm{RM}=$ razón de momios de la prevalencia

Fuente: elaboración propia con base en datos de las encuestas realizadas a los refugiados guatemaltecos en CH82, Q R89 y CAQ R96

12 a 35 meses es el que, a lo largo del periodo, ha presentado la mayor prevalencia de bajo peso para la edad, con un riesgo significativo de desnutrición de I, II y III grados en $\mathrm{CH} 82$ ( $\mathrm{RMP}=12.2,27.0$ y 5.2, respectivamente), desnutriciónde I y II en QR89 (RMP= 6.6 y 2.1, respectivamente) y de I grado en $\mathrm{CAQR} 96(\mathrm{RMP}=4.0)$. El grupo de 36 a 71 meses no tiene riesgo significativo de desnutrición de III grado en ninguno de los años; sin embargo, presenta prevalencias importantes de I y II grados en $\mathrm{CH} 82$ y QR89 (RMP= I grado 11.5 y 5.4; II grado 14.5 y 1.4, respectivamente). Por último, en el grupo de menores de un año se obtuvieron las menores prevalencias de bajo peso para la edad y, a excepción de los casos donde se presentó desnutrición de I y II gradosen $\mathrm{CH} 82$ (RMP= 2.9 y 3.7, respectivamente), no se encontraron otras diferencias significativas.

Los cuadros IV y V comprenden la comparación en el mismo año (1989) de la población que permaneció en Chiapas y de la que fue reubicada al estado de Quintana Roo en 1984, según los índices peso para la edad y talla para la edad en población total y por grupos de edad.
El cuadro IV permite observar cómo en los menores de seis años del estado de Chiapas existía 1.8 veces mayor riesgo de que se presentara desnutrición de II grado, en comparación con los niños refugiados en Quintana Roo. En cuanto al I y el III grado no se encontraron diferencias significativas entre los niños de ambos estados. En el grupo de menores de un año se observa mayor riesgo de desnutrición de I y II grados en CH89 comparado con Quintana Roo en el mismo año (RMP $=1.4$ y 2.0, respectivamente). Respecto a los niños de 12 a 35 meses no se encontraron diferencias significativas. En el grupo de 36 a 71 meses existe 2.6 veces mayor riesgo de padecer desnutrición de II grado en $\mathrm{CH} 89$, contrario a lo que sucede con la desnutrición de I grado y el estado de nutrición normal, en donde las prevalencias más altas las tienen los niños de Quintana Roo. En ningún grupo de edad se encontraron diferencias significativas de desnutrición de III grado.

El cuadro $\mathrm{V}$ muestra igualmente las diferencias entre Chiapas y Quintana Roo pero con base en la comparación del índice de talla para la edad del grupo de 


\section{Cuadro III}

\section{Estado nutricional de la población refugiada guatemalteca menor de seis años, por GRUPOS de edad SEGÚN EL INDICADOR PESO PARA LA EDAD. COMPARACIÓN POR GRAdO dE DESNUTRICIÓN}

\begin{tabular}{|c|c|c|c|c|c|c|c|c|c|c|c|}
\hline \multirow[t]{2}{*}{ Estudio } & \multicolumn{2}{|c|}{ Normal } & \multicolumn{3}{|c|}{ I grado } & \multicolumn{3}{|c|}{ II grado } & \multicolumn{3}{|c|}{ III grado } \\
\hline & $\%$ & RMP & $\%$ & RMP & ТС $95 \%$ & $\%$ & RMP & IC $95 \%$ & $\%$ & RMP & IC $95 \%$ \\
\hline \multicolumn{12}{|l|}{$<$ de 12 meses } \\
\hline $\mathrm{CH} 82$ & 15.3 & 1.00 & 34.0 & 2.86 & $1.57-5.32$ & 40.3 & 3.74 & $2.0-6.90$ & 10.4 & 0.64 & ns \\
\hline QR89 & 55.4 & 1.00 & 33.1 & 0.40 & $0.29-0.55$ & 9.0 & 0.08 & $0.05-0.13$ & 2.4 & 0.02 & $0.01-0.04$ \\
\hline CAQR96 & 47.8 & 1.00 & 43.5 & 0.84 & ns & 8.7 & 0.10 & $0.01-0.62$ & 0.0 & 0.00 & $0.00-0.26$ \\
\hline
\end{tabular}

\begin{tabular}{|c|c|c|c|c|c|c|c|c|c|c|c|}
\hline $\mathrm{CH} 82$ & & & & 127 & & & & & & & \\
\hline & & & & & & & & & & & \\
\hline Q R89 & 13.7 & 1.00 & 51.2 & 6.63 & $4.36-10.20$ & 30.7 & 2.08 & $1.82-4.37$ & 4.4 & 0.29 & $0.14-0.58$ \\
\hline CAQR96 & 24.0 & 1.00 & 56.0 & 4.03 & $2.27-7.20$ & 20.0 & 0.79 & ns & 0.0 & 0.00 & $0.00-0.10$ \\
\hline
\end{tabular}

\begin{tabular}{|c|c|c|c|c|c|c|c|c|c|c|c|}
\hline $\mathrm{CH} 82$ & 58 & 100 & & 1151 & $670-2066$ & 471 & 1455 & $810 \quad 2607$ & & & $m$ \\
\hline QR89 & 22.0 & 1.00 & 60.1 & 5.37 & $4.29-6.72$ & 160 & 141 & $112-179$ & 19 & 007 & \\
\hline CAQR96 & 20.2 & 1.00 & 60.3 & 5.99 & $4.00-9.00$ & 19.0 & 0.93 & ns & 0.4 & 0.01 & $0.00-0.09$ \\
\hline
\end{tabular}

\begin{tabular}{|c|c|c|c|c|c|c|c|c|c|c|c|}
\hline CH82 & 6.9 & 1.00 & 36.1 & 7.64 & $5.47-10.83$ & 46.6 & 11.82 & $8.48-16.70$ & 10.4 & 1.57 & $1.06-2.34$ \\
\hline QR89 & 28.1 & 1.00 & 52.0 & 2.78 & $2.38-3.26$ & 17.4 & 0.54 & $0.45-0.65$ & 2.5 & 0.07 & $0.05-0.09$ \\
\hline CAQR96 & 22.9 & 1.00 & 58.1 & 4.67 & $3.42-6.38$ & 18.8 & 0.78 & ns & 0.2 & 0.01 & $0.00-0.05$ \\
\hline
\end{tabular}

RMP = razón de momios de la prevalencia ns $=$ no significativo

Fuente: elaboración propia con base en datos de las encuestas realizadas a los refugiados guatemaltecos en CH82, Q R89 y CAQ R96

menores de seis años. La prevalencia de retraso en el crecimiento expresado por la talla en Chiapas llega a $85 \%$ contra $75 \%$ de Quintana Roo. El riesgo de déficit de talla (pequeños y achaparrados) fue significativamente mayor en Chiapas que en Quintana Roo (RMP= 1.28 y 1.36, respectivamente). Si se analizan los datos por grupos de edad, en el de menores de un año en CH89 existe 2.3 veces mayor riesgo de ser pequeños que en QR89, y una mayor probabilidad en QR89 de tener una talla, normal o de ser achaparrados (RMP= 0.51 y 0.22 , respectivamente). En el grupo de 12 a 35 meses no se encontraron diferencias significativas en cuanto a déficit de talla, y en el grupo de niños de 36 a 71 meses los datos indican un riesgo 1.8 veces mayor de ser achaparrados en CH89 que en Quintana Roo.

\section{Características de los programas de ayuda alimentaria}

Ala llegada de los primeros refugiados, las poblaciones campesinas mexicanas, algunas organizaciones civiles y trabajadores de salud de diversas instituciones en el estado de Chiapas, ofrecieron ayuda solidaria. Desde principios de 1982, el Hospital Regional "C" de la Secretaría de Salud, en Comitán, Chiapas, había dado a conocer la grave situación de salud de los refugiados. En un corto periodo se incrementaron las hospitalizaciones de guatemaltecos, por lo que su capacidad de atención fue totalmente rebasada. ${ }^{*}$

Poco después la atención a los refugiados se institucionalizó, de tal modo que se desarrollaron diversos programas. Para su diseño y ejecución, a mediados de 1982 se firmaron convenios entre el gobierno mexicano y tres agencias de la Organización de las Naciones Unidas (ONU): el ACNUR, el Fondo de las Naciones Unidas para la Infancia (UNICEF) y el Programa Mundial de Alimentos (PMA). En los convenios se estipuló que las agencias de la ONU proporcionarían los recursos y la asesoría técnica; la ejecución y la ad-

* Hospital General "C" de la Secretaría de Salud en Comitán, Chiapas. Informe presentado a UNICEF sobre la situación del Hospital de Comitán en relación con los refugiados guatemaltecos. México, D.F.: Secretaría de Salud, 1982. Documento no publicado. 


\section{Cuadro IV \\ Estado NUtRicional de La población Refugiada guatemalteca menor de SEIS años SEgún EL INDICAdor PeSO PARA LA EDAD. Comparación POR ESTUdio (CH89 vs QR89)}

Estudio

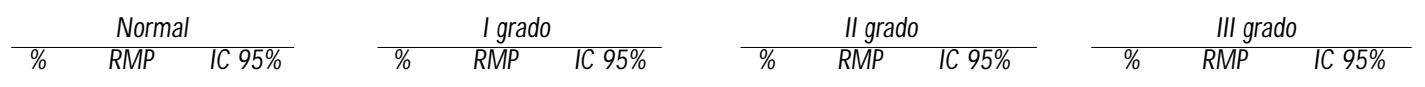

$<$ de 12 meses

\begin{tabular}{cccccccccccccc} 
CH89 & 40.7 & 0.55 & $0.40-0.77$ & 41.4 & 1.43 & $1.01-2.01$ & 16.4 & 1.98 & $1.18-3.33$ & 1.4 & 0.59 & ns \\
\hline Q R89 & 55.4 & 1.00 & - & 33.1 & 1.00 & - & 9.0 & 1.00 & - & 2.5 & 1.00 & -
\end{tabular}

12 a 35 meses

\begin{tabular}{cccccccccccccc} 
CH89 & 12.5 & 0.90 & ns & 52.9 & 1.07 & ns & 32.2 & 1.07 & ns & 2.4 & 0.53 & ns \\
\hline Q R89 & 13.6 & 1.00 & - & 51.2 & 1.00 & - & 30.7 & 1.00 & - & 4.5 & 1.00 & -
\end{tabular}

36 a 71 meses

\begin{tabular}{lllllllllllll} 
CH89 & 11.2 & 0.45 & $0.33-0.61$ & 53.7 & 0.77 & $0.62-0.95$ & 33.2 & 2.62 & $2.02-3.39$ & 1.9 & 1.02 & ns \\
\hline Q R89 & 22.0 & 1.00 & - & 60.2 & 1.00 & - & 16.0 & 1.00 & - & 1.8 & 1.00 &
\end{tabular}

Total

\begin{tabular}{cccccccccccccc} 
CH89 & 17.4 & 0.56 & $0.46-0.68$ & 51.0 & 0.96 & ns & 29.6 & 1.84 & $1.55-2.25$ & 2.0 & 0.72 & ns \\
\hline Q R89 & 28.1 & 1.00 & - & 52.0 & 1.00 & - & 17.4 & 1.00 & - & 2.5 & 1.00 & -
\end{tabular}

RMP = razón de monios de la prevalencia $\mathrm{ns}=$ no significativo

Fuente: elaboración propia con base en datos de las encuestas realizadas a los refugiados guatemaltecos en CH 89 y Q R89

\section{Cuadro V}

Estado nUtricional de La poblaciónm Refugiada guatemalteca Menor de SEIS años, SEgún el indicador talla para la edad. Comparación por estudio (CH89 vs QR89)

\begin{tabular}{|c|c|c|c|c|c|c|c|c|c|}
\hline \multirow[t]{2}{*}{ Estudio } & \multicolumn{3}{|c|}{ Normal } & \multicolumn{3}{|c|}{ Pequeños } & \multicolumn{3}{|c|}{ Achaparrados } \\
\hline & $\%$ & RMP & IC $95 \%$ & $\%$ & RMP & IC 95\% & $\%$ & RMP & IC 95\% \\
\hline$<$ de $12 \mathrm{me}$ & & & & & & & & & \\
\hline $\mathrm{CH} 89$ & 40.4 & 0.51 & $0.36-0.71$ & 58.6 & 2.29 & $1.64-3.21$ & 1.1 & 0.22 & $0.05-0.81$ \\
\hline QR89 & 57.2 & 1.00 & - & 38.1 & 1.00 & - & 4.7 & 1.00 & - \\
\hline
\end{tabular}

12 a 35 meses

\begin{tabular}{ccccccccccc} 
CH89 & 13.2 & 0.78 & ns & 76.9 & 1.27 & ns & 9.9 & 0.86 & ns \\
\hline Q R89 & 16.4 & 1.00 & - & 72.3 & 1.00 & - & 11.3 & 1.00 & -
\end{tabular}

36 a 71 meses

\begin{tabular}{|c|c|c|c|c|c|c|c|c|c|}
\hline $\mathrm{CH} 89$ & 5.4 & 0.37 & $0.24-0.57$ & 71.9 & 0.95 & ns & 22.7 & 1.85 & $1.39-2.46$ \\
\hline Q R89 & 13.3 & 1.00 & - & 73.0 & 1.00 & - & 13.7 & 1.00 & - \\
\hline
\end{tabular}

Total

\begin{tabular}{|c|c|c|c|c|c|c|c|c|c|}
\hline $\mathrm{CH} 89$ & 15.1 & 0.52 & $0.42-0.65$ & 71.1 & 1.28 & $1.09-1.52$ & 13.7 & 1.36 & $1.08-1.75$ \\
\hline Q R89 & 24.5 & 1.00 & - & 64.5 & 1.00 & - & 11.0 & 1.00 & - \\
\hline
\end{tabular}

RMP = razón de monios de la prevalencia ns= no significativo

Fuente: elaboración propia con base en datos de las encuestas realizadas a los refugiados guatemaltecos en CH89 y Q R89 
ministración de los programas quedarían bajo la responsabilidad del gobierno mexicano representado por la COMAR.

En los 14 años transcurridos se pueden distinguir tres épocas en la ayuda alimentaria: la primera, de 1982 a 1984, cuando tuvo una perspectiva de emergencia, con dos componentes: la distribución de alimentos familiares* financiada por el PMA y el Programa de Atención a Grupos Vulnerables (PAGV) que estuvo financiado por el UNICEF., Se consideró como vulnerables a las mujeres embarazadas y/o lactando y a los niños y niñas menores de seis años, que representaban 38\% de la población total. Se proporcionaban diariamente, de manera diferencial según edad y estado de nutrición, tres preparaciones básicas: atole, papillas y leche. Se diseñó un sistema de diagnóstico y vigilancia del estado de nutrición que utilizó el indicador peso para la edad y la clasificación de Gómez como criterio diagnóstico. Este sistema permitió identificar a los desnutridos graves y en riesgo, los cuales requerían mayor cantidad de alimentos. Se administraron suplementos de vitaminas y minerales y terapia de rehidratación oral. Este programa llegó a operar en los nueve campamentos de mayor tamaño en el estado de Chiapas, en los que la población total sumaba 19000 refugiados. $\$$

La segunda época, que va de 1984 a 1989, se caracterizó por esquemas de ayuda alimentaria diferentes para la población que permaneció en Chiapas de la que se reubicó en otros sitios. En Chiapas se suspendió el PAGV y continuó la dotación de alimentos familiares. Para los reubicados, se modificaron los criterios del programa al incluir sólo a los menores de seis años como destinatarios y utilizar el indicador peso para la talla en el diagnóstico. Se establecieron dos estrategias para este Programa: a) el Programa de Alimentación Complementaria (PAC), que consistía en entregar a las familias quincenalmente $1 \mathrm{~kg}$ de cereal, $1 / 2 \mathrm{~kg}$ de frijol, $1 / 21$ de aceite y $1 \mathrm{~kg}$ de azúcar por cada menor de seis años, y b) el Programa de Alimentación Terapéutica (PAT) para proporcionar alimentos preparados -atole y papilla- a los menores de seis años que pre-

\footnotetext{
* La alimentación familiar se estructuró en raciones diarias per cápita de la siguiente manera: $300 \mathrm{~g}$ de maíz, $100 \mathrm{~g}$ de frijol, 50 $\mathrm{g}$ de azúcar, $40 \mathrm{~g}$ de arroz o avena, $60 \mathrm{~g}$ de zanahoria o papa, $25 \mathrm{~g}$ de aceite, $40 \mathrm{~g}$ de huevo, queso o pescado seco.

₹ Peláez ML, Torre P. Informe a UNICEF sobre la situación de los refugiados guatemaltecos en Chiapas: propuesta para la atención nutricional de emergencia. México, D.F.: Secretaría de Salud, 1982 (mimeo).

§ Peláez ML, Torre P. Informe de la visita de supervisión al Programa de Alimentación a Grupos Vulnerables. Chiapas: UNICEF, SSA, 1984 (mimeo).
}

sentaran un peso para la talla menor o igual a $70 \%$ del esperado. La distribución de alimentos familiares se redujo progresivamente a medida que aumentaba la producción local.*

La tercera época, de 1989 a 1996, se fundamenta en la encuesta realizada en 1989, en cuyas conclusiones se señala que no existe diferencia en el estado de nutrición de los refugiados respecto a la población mexicana. Así, dadas las condiciones de los que se quedaron en Chiapas, se mantuvo la alimentación familiar, mientras que para los reubicados en Campeche y Quintana Roo se limitó en función de la autosuficiencia, y el PAGV quedó restringido al PAT.

\section{Discusión}

Este trabajo presenta la comparación del estado de nutrición de diferentes generaciones de niños menores de seis años de la población refugiada guatemalteca a lo largo de su estancia en México, lo cual da cuenta del impacto que tuvieron los cambios en sus condiciones de vida y de salud como resultado, principalmente, de los programas de ayuda destinados a apoyar a estos grupos, tanto para la situación de emergencia como para la estabilización de la situación de la población.

Si bien no se puede asegurar que la población refugiada estudiada en 1982, 1989 y 1996 es la misma, se puede partir del supuesto de que la población menor de seis años estudiada en 1982 era representativa del resto de los refugiados asentados en la zona de la selva chiapaneca y que esta población es la que se reubicó en los campamentos de Campeche y Quintana Roo en 1984 y permaneció más o menos estable hasta 1996. La encuesta CH82 evaluó a la generación nacida en Guatemala entre 1976 y 1982, que sufrió las consecuencias del fenómeno migratorio. El segundo estudio, $\mathrm{CH} 89$ y QR89 valoró a la generación 1983-1989, que nació en México aunque vivió en circunstancias diferentes ya que la reubicación estableció diferentes condiciones para unos y para otros y, por último, el estudio de 1996 valoró a la generación nacida entre 1990 y 1996 que fue reubicada en los estados de Campeche y Quintana Roo.

De acuerdo con los resultados se puede concluir que los programas de ayuda alimentaria son muy útiles en casos de emergencia, principalmente atenuando los estados más graves de desnutrición y la mortalidad. Respecto a esta última, hubiera resultado de gran interés conocer su evolución a lo largo del pe-

\footnotetext{
* Berry A, Torre P. Informe de la misión sobre nutrición de la sede de ACNUR en Ginebra. Ginebra: ACNUR, 1984 (mimeo).
} 
riodo; sin embargo, los únicos datos disponibles para esta población se recabaron en Chiapas en 1982 y 1983. Dichos datos muestran una tasa anualizada de mortalidad, observada al momento de su llegada a México (1982), de 477 defunciones por 1000 menores de seis años, y cuatro meses después, en el periodo posterior a la instalación del Programa, se redujo a 103 defunciones por 1000 menores de seis años. Esto parece indicar que el Programa, a 16 semanas de haberse iniciado su operación, tuvo un gran impacto sobre la mortalidad de los niños; desafortunadamente no se cuenta con información reciente de la mortalidad en dicha población.

En lo referente al estado nutricional, los programas de largo plazo casi lograron eliminar la desnutrición de III grado; no obstante, la de I y II grados siguió siendo un problema prevalente en estos grupos, sobre todo porque las políticas de la ONU establecen que la ayuda alimentaria y de salud para las poblaciones refugiadas no debe generar mejores condiciones que las de la población del país receptor. ${ }^{10}$

Dentro del grupo de menores de seis años, los niños mayores de un año son los que tienen mayor riesgo de desnutrición ya que los menores de un año se encuentran protegidos, seguramente por la lactancia materna.

Por otra parte al comparar a la población de Chiapas con la de Quintana Roo en 1989, se encontró que en Chiapas había un mayor deterioro del estado nutricional, situación que se explica por las diferencias cuantitativas y cualitativas de los programas instaurados en cada zona. En Chiapas el PAGV terminó en 1985, mientras que en Campeche y Quintana Roo en esa misma fecha se inició y continuó hasta 1989. En relación con la inversión en programas para cada una de las regiones el presupuesto per cápita anual presentó grandes diferencias en 1986; en Chiapas fue de 49714 pesos corrientes; en Campeche, de 110153 pesos, y en Quintana Roo, de 185011 pesos. Por otra parte, en Campeche y Quintana Roo se desarrollaron proyectos productivos que dotaron a la población de 2645 hectáreas de tierras para el cultivo de productos básicos y para la comercialización, lo cual les daba una mayor estabilidad y cierta independencia en relación con los programas de ayuda alimentaria. Esto se corrobora con el hecho de que, aunque en 1989 se suspendió el PAGV, el estado de nutrición de la población de Campeche y Quintana Roo no presentó cambios respecto a 1996.

Finalmente, se puede concluir que los programas de ayuda alimentaria directa tienen gran importancia para aliviar el hambre crónica y aguda, cuando los individuos no disponen de la capacidad para cubrir sus necesidades por razones que se salen de su alcance, como fue el caso de los refugiados guatemaltecos.

\section{Agradecimientos}

Se agradece a los doctores Antonio Villa, por la asesoría que brindó para el anáisis estadístico, y Adolfo Chávez, por su entusiasmo y apoyo para la publicación de este trabajo.

\section{Referencias}

1. A guayo S, Cabral A. Guatemala: el evangelio del genocidio. N exoS 1983; 9:35-41.

2. Ciencia y Tecnología para Guatemala. Crisis en Centroamérica y refugiados guatemaltecos en México. Guatemala: CITGUA, 1985; 5:26.

3. Torre P. Evaluación del estado nutricional y su relación con la mortalidad en los menores de seis años en la población de refugiados guatemaltecos ubicada en México (tesis). México,D.F.: Universidad Iberoamericana, 1984.

4. Torre P. N utrición y refugiados. Estudios fronterizos 1988;7:81-103.

5. Torre P. Estado de nutrición y mortalidad en los guatemaltecos menores de seis años refugiados en México. En: Health care for displaced persons: An International Symposium. Georgetown University Medical Center; 1988 Dic 4-7;W ashington, D.C., USA.

6. Gómez S. Desnutrición. Bol Med Hosp Infant 1946;3:543-551.

7. Avila A, Shamah T, Chávez A. Encuesta Nacional de Alimentación y Nutrición en el Medio Rural 1996. México, D.F.: IN N SZ, 1996.

8.Ysunza A. Encuesta 0 axaqueña de N utrición. En: Madrigal H, ed. Agricultura, alimentación y nutrición en México: década de los ochenta. México, D.F.: IN N SZ/O PS/O MS, 1996.

9. De Ville C, Seaman J, Geijer U. El manejo de las emergencias nutricionales en grandes poblaciones. Publicación científica 444. Washington, D.C.:O rganización Panamericana de la Salud, O rganización Mundial de la Salud, 1983

10. United $\mathrm{N}$ ations High Commissioner for Refugees. Handbook for emergencies. Ginebra: UN HCR, 1982. 